\title{
Correction to "TD-19, an Erlotinib Derivative, Induces Epidermal Growth Factor Receptor Wild-Type Nonsmall-Cell Lung Cancer Apoptosis through CIP2A-Mediated Pathway"
}

The first author of the above article [Chao T-T, Wang C-Y, Lai C-C, Chen Y-:L, Tsai Y-T, Chen P-T, Lin H-I, Huang Y-CT, Shiau C-W, Yu C-J, and Chen K-F (2014) J Pharmacol Exp Ther 351(2):352-358. DOI: https://doi.org/10.1124/jpet.114.215418], recently identified the following errors:

In Figure 5 the authors misplaced (Fig 5A, left panel and Fig 5C, left panel) which appeared in another published article (Lung Cancer. 2014 ;85(2):152-60. doi: 10.1016/j. lungcan.2014.05.024).

The results of Figure 5 are consistent with the conclusions published in the original article.

The corrected figure appears below.

A

H460

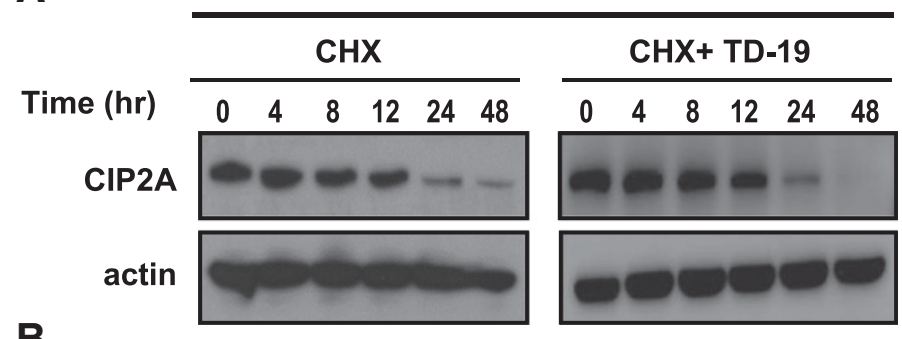

B

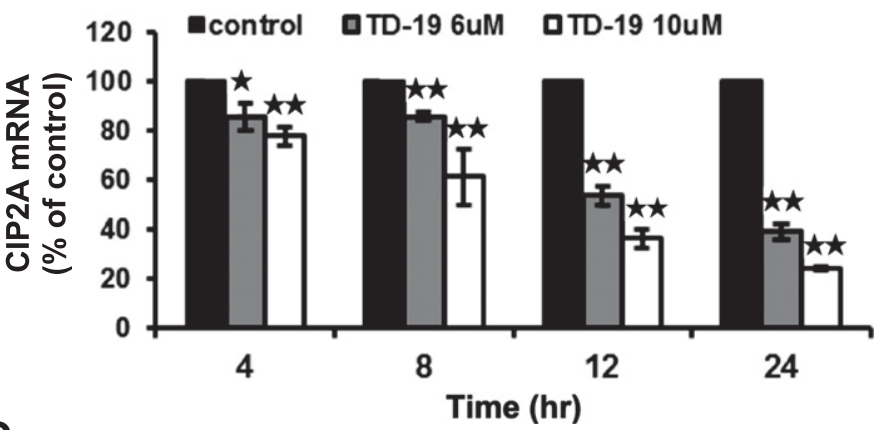

C
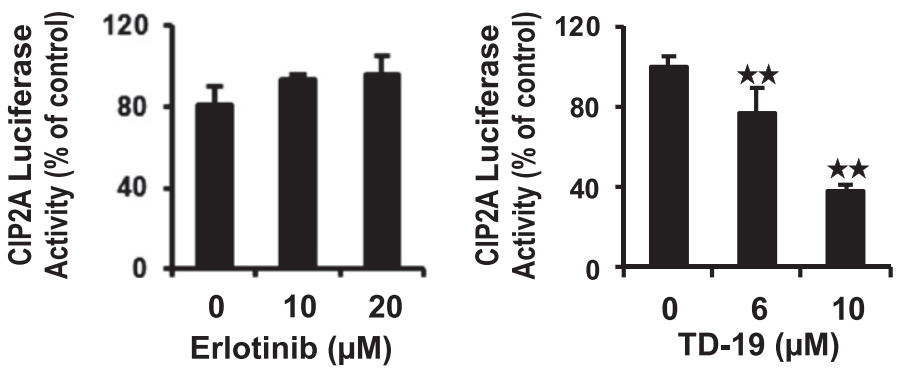
In Figure 6B the western of CIP2A, p-AKT, AKT and actin of nos. 1, 2, and 3 were similar to nos. 9,8 , and 7 .

The results of Figure 6 are consistent with the conclusions published in the original article.

The corrected figure appears below.

A

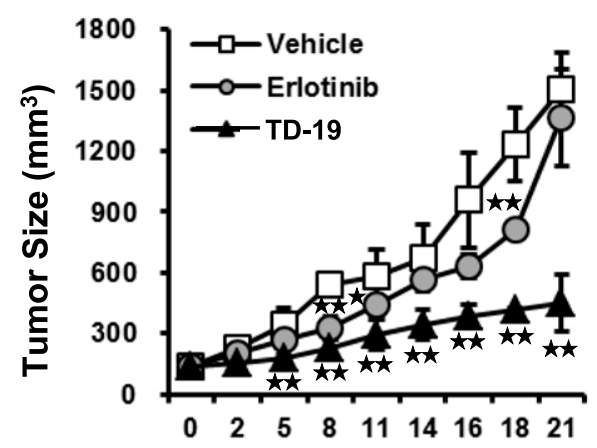

Days of Treatment

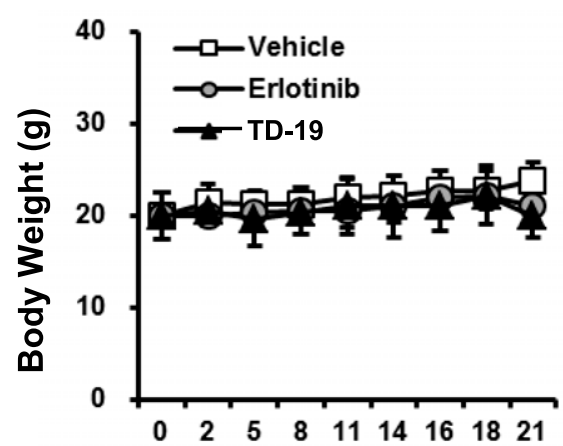

Days of Treatment
B

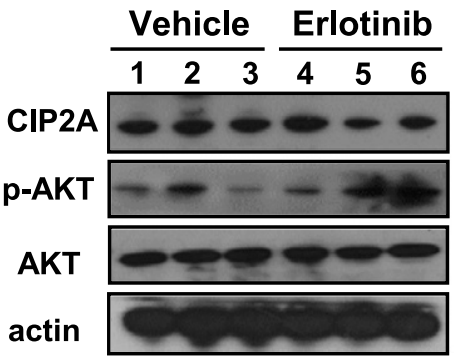

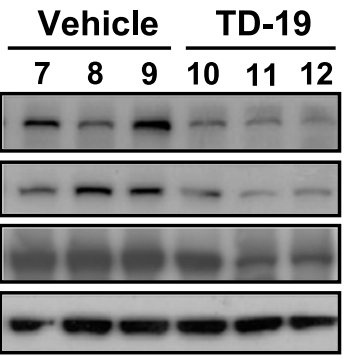

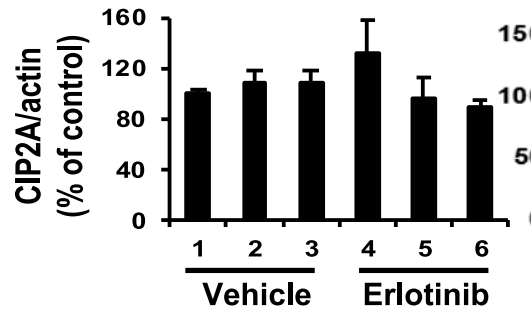

so

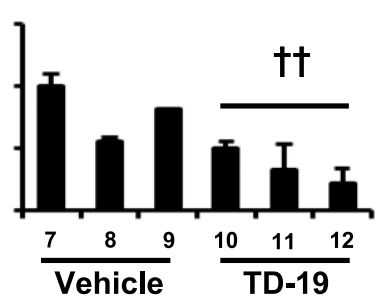

C

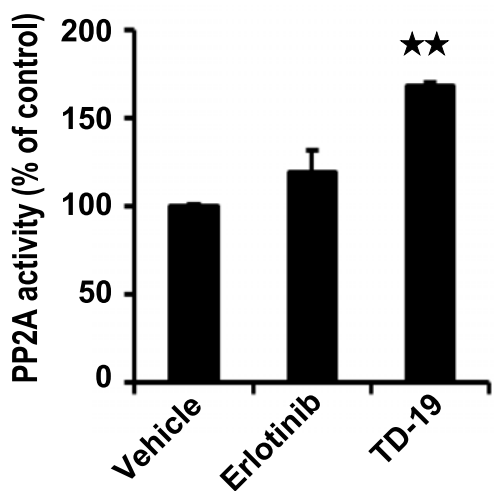

D

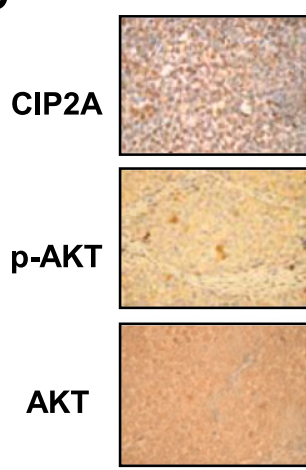

\section{Erlotinib}
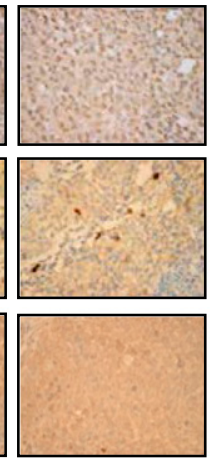

TD-19

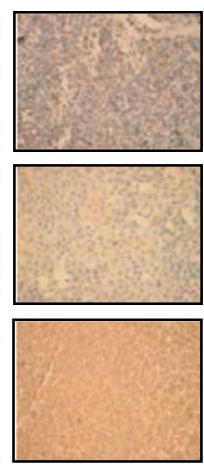

QVehicle QErlotinib $\square T D-19$

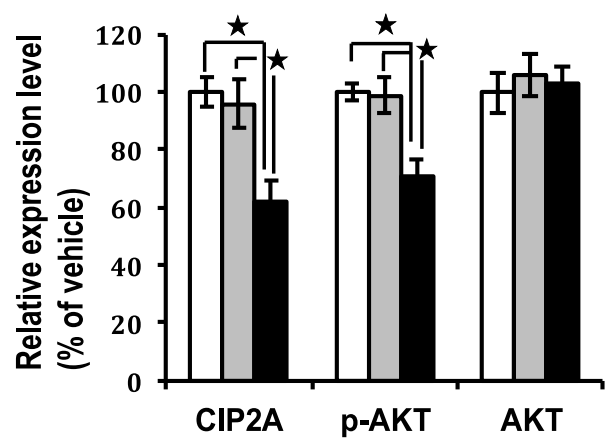

Figure 6. Effect of erlotinib or TD-19 on H460 xenograft tumor growth in nude mice. (A) Mice were treated with vehicle, erlotinib or TD-19 p.o at $10 \mathrm{mg} / \mathrm{kg}$ daily for 3 weeks. TD-19 inhibited tumor growth by approximately $80 \%$ (left). There was no difference in body weight (right). Data are shown as mean \pm SD. $\mathrm{n}=6,{ }^{*}, P<0.05$; **, $P<0.01$. Statistical analyzed by ANOVA. (B) Western blot analysis of CIP2A, p-AKT and AKT in H460 tumors. Ratio of CIP2A to actin is shown below each western blot data set. Immunoblots were quantitated using VisionWork LS software. $\dagger \dagger$ Represents the $p$ value $<0.01$ when comparing the mean percentage of the TD19-treated group (no. 10,11,12) with the mean percentage of the vehicle group (no.7, 8,9 ) by ANOVA. (C) Analysis of PP2A activity in tumors. Data are shown as mean \pm SD. $\mathrm{n}=6$; *, $P<0.05$; **, $P<0.01$. All data are representative of three independent experiments. Statistically analyzed by ANOVA. (D) Immunohistochemical stain and quantitative analyses of CIP2A, p-AKT and AKT in H460 xenografts tumors (400X magnification). Data are shown as mean $\pm \mathrm{SD} . \mathrm{n}=6 ; *, P<0.05$. Statistical analyzed by ANOVA. 